\section{Alteração tireoidiana: um fator de risco associado à depressão pós-parto?}

\section{Thyroid dysfunction: a risk factor associated with post-partum depression?}

\section{Abstract}

Objectives: to investigate the prevalence of postpartum depression and its correlation with thyroid dysfunction in mothers.

Methods: a descriptive, cross-cutting observational study carried out among 292 women between the 31 st and $180^{\text {th }}$ days post partum, attending public health units in the Metropolitan Region of Vitoria, in the State of Espirito Santo, Brazil. The sample was analyzed for socio-demographic and clinical-obstetric characteristics. A depressive disorder was defined by a score greater than or equal to twelve on the Edinburgh Post-Partum Depression Scale (EPDS). The thyroid was assessed using serum dosages of thyroid stimulating hormone, (TSH), free thyroxin (T4 free) and anti-peroxidase antibodies (TPO). Evaluation of the results was carried out using descriptive analysis and the $\chi^{2}$ test, with a level of significance of $5 \%$.

Results: 115 women (39.4\%) scored higher than 12 on the EPDS and were thereby deemed to be depressed; $117(60.6 \%)$ scoring lower than 12 were considered not to be depressed. The prevalence of post-partum depression in the group with thyroid dysfunction was $36 \%$ and $40 \%$ in the group without thyroid dysfunction. There was no statistically significant difference in the frequency of depression between patients with or without thyroid dysfunction $\left(\chi^{2}=0.131 ; p=0.717\right)$.

Conclusions: the frequency of PPD was high but no association was observed between post-partum depression and thyroid dysfunction.

Key words Depression, postpartum, Postpartum thyroiditis
Gustavo Enrico Cabral Ruschi 1

Antônio Chambô Filho 2

Valmir José de Lima 3

Sue Yazaki-Sun 4

Eliana Zandonade 5

Rosiane Mattar 6

1,3,4,6 Programa de Pós-Graduação em Obstetrícia. Escola Paulista de Medicina. Universidade Federal de São Paulo (UNIFESP/EPM). Rua Napoleão de Barros 875, Vila Clementino. CEP: 04024-002. São Paulo, SP, Brasil. E-mail: gruschi@terra.com.br

2 Departamento de Ginecologia e Obstetrícia. Escola de Ciências Superiores da Santa Casa de Misericórdia do Espírito Santo. Vitória, Espírito Santo, Brasil.

5 Programa de Pós-Graduação em Saúde Coletiva da Universidade Federal do Espírito Santo (UFES), Vitória, Espírito Santo, Brasil.

\section{Resumo}

Objetivos: estudar prevalência de depressão pósparto e sua correlação com alterações tireoidianas maternas.

Métodos: estudo observacional descritivo transversal realizado com 292 mulheres entre 31 e 180 dias após o parto, atendidas em unidades de saúde pública da Região Metroplitana de Vitória, Espirito Santo, Brasil. Analisou-se a amostra segundo características sociodemográficas e clínico-obstétricas. Definiu-se transtorno depressivo pelo escore igual ou superior a 12 na Escala de Depressão Pós-Parto de Edimburgo (EPDS). A avaliação tireoidiana foi realizada por dosagens séricas de hormônio tireo-estimulante (TSH), tiroxina livre (T4 livre) e dos anticorpos anti-peroxidase (TPO). Para avaliação dos resultados utilizamos técnicas de análise descritiva e teste do $\chi^{2}$, adotando nível de significância de $5 \%$.

Resultados: 115 mulheres (39,4\%) apresentaram escores iguais ou superiores a 12 na EPDS, sendo consideradas deprimidas; 117 (60,6\%), com escores inferiores a 12, foram consideradas não deprimidas. A prevalência de depressão pós-parto no grupo com alterações tireoidianas foi de $36 \%$ e no grupo sem alterações tireoidianas foi de $40 \%$. Não houve diferença estatisticamente significante na frequência de depressão entre as pacientes com e sem alterações tireoidianas $\left(\chi^{2}=0,131 ; p=0,717\right)$.

Conclusões: a frequência de DPP foi elevada, não sendo observada associação entre depressão pós-parto e alterações tireoidianas.

Palavras-chave Depressão pós-parto, Tireoidite pósparto 


\section{Introdução}

O período pós-parto, considerado uma das vivências mais complexas da experiência humana, ${ }^{1}$ caracteriza-se por uma vulnerabilidade emocional que, associada às mudanças fisiológicas, psicológicas, sociais e culturais propicia o aparecimento de transtornos mentais maternos.

A depressão, um dos transtornos mentais mais frequentes após o parto, é considerada sério e atual problema de Saúde Pública, pela sua alta prevalência e pelo impacto negativo que exerce na estrutura e dinâmica familiar. Estudos nacionais descrevem prevalências da depressão pós-parto (DPP) variando de 12 a $19 \%,{ }^{2-5}$ dados compatíveis com a literatura internacional.6,7

A depressão pós-parto, por não diferir qualitativamente da depressão que ocorre em outras fases da vida, 8 durante muitos anos não foi considerada entidade clínica psiquiátrica distinta. Somente em 1994 a Associação Psiquiátrica Americana (APA) reconheceu a DPP como um grupo específico de transtorno do humor, incluindo-a no "Manual Diagnóstico e Estatístico de Transtornos Mentais (DSM)" e definindo-a como a depressão que tem início nas quatro primeiras semanas após o término da gestação, com duração de pelo menos duas semanas e que apresenta no mínimo cinco dos seguintes sintomas: humor deprimido, anedonia, mudanças do apetite ou peso, insônia ou hipersonia, agitação ou retardo psicomotor, fadiga, sentimento de culpa ou inutilidade, capacidade diminuída de concentração, raciocínio ou indecisão, e pensamentos recorrentes de morte. 9,10

A complexidade dos critérios diagnósticos da APA limitam sua utilização por profissionais não especializados em saúde mental. Com a adoção de instrumentos padronizados, como as escalas de depressão, que traduzem o fenômeno clínico em informações objetivas e quantitativas, tornou-se mais fácil a suspeita diagnóstica dos quadros depressivos por profissionais que atuam nos níveis primários de atenção à saúde. ${ }^{11,12}$

Estudos epidemiológicos sobre depressão pósparto sugerem uma multiplicidade de fatores de risco envolvidos na sua gênese, entre os quais as endocrinopatias. ${ }^{13-16}$

Entre as endocrinopatias, destacam-se as alterações tireoidianas que podem ocorrer até 12 meses após o parto, ${ }^{17}$ principalmente na forma de tireoidite auto-imune, precipitadas por alterações imunológicas do puerpério presentes em 5 a $9 \%$ da população feminina,18-22 cuja forma clínica clássica se caracteriza por uma fase tireotóxica inicial (segundo ao quarto mês), seguida pela fase hipotireoidea (quarto ao oitavo mês), com posterior retorno ao estado eutireoidiano. ${ }^{17}$ Das doenças endócrinas, as alterações tireoidianas são as que mais têm sido pesquisadas no sentido de se identificar associação à depressão pós-parto.

A depressão e as alterações da tireóide no pósparto estão relacionadas à qualidade de vida da mulher e do recém-nascido e têm influência social e econômica não só para aquelas famílias, mas também para a sociedade como um todo. Julgando importante o conhecimento da depressão e seus fatores associados, este estudo tem como objetivo determinar a prevalência de sintomas depressivos e das alterações tireoidianas no período pós-parto, na tentativa de estabelecer associação entre as duas entidades, permitindo a adoção de medidas preventivas, de acompanhamento e assistência perinatais, importantes na proteção do binômio mãe-filho, com redução dos efeitos adversos sobre o desenvolvimento cognitivo e emocional da criança.

\section{Métodos}

Foi realizado estudo observacional descritivo transversal com mulheres em pós-parto, atendidas nos Ambulatórios de Ginecologia e Obstetrícia da Unidade de Saúde da Família de Maruípe, bairro pertencente ao município de Vitória e da Unidade Regional de Saúde de Feu Rosa, no município de Serra, região metropolitana da Grande Vitória, estado do Espírito Santo, Brasil.

O cálculo da amostra baseou-se na fórmula de teste de diferenças entre duas proporções, considerando o percentual de mulheres com depressão no grupo de disfunção tireoidiana de $38 \%$ e no grupo sem disfunção o percentual de $9,5 \%, 23$ poder de $80 \%$ e nível de significância de $5 \%$. O valor mínimo de amostra calculado foi de 160 pacientes.

Foram elegíveis, para esse estudo, 292 mulheres que procuraram atendimento nos referidos ambulatórios, em um período de dois anos de coleta de dados (junho de 2004 a maio de 2006), e que preenchiam os seguintes critérios de inclusão: tempo de pós-parto entre 31 e 180 dias; gestação única cuja resolução se deu com 34 a 42 semanas e idade materna entre 15 e 45 anos. Foram excluídas as pacientes com história de natimorto ou neomorto na atual gestação, idade gestacional inferior a 34 semanas, complicações neonatais na atual gestação, histórico de uso de drogas psicoativas ou tratamento psiquiátrico prévio e histórico de doenças autoimunes (lupus eritematoso, artrite reumatóide, diabetes mellitus). 
Para identificar o perfil da população estudada, foi elaborada entrevista semi-estruturada, padronizada, aplicada pelo pesquisador, em ambiente privativo, constando de informações sociodemográficas, fatores associados ao comportamento e hábito de vida e dados clínico-obstétricos e neonatais, assim definidos: idade (aferida em anos completos); estado civil: (solteira, casada, divorciada, conforme certidão legal); cor; escolaridade (grau máximo de instrução alcançado, a considerar: nenhum grau de instrução, ensino fundamental incompleto ou completo, ensino médio incompleto ou completo, ensino superior); renda familiar média (somatória do número de salários mínimos recebidos pelos membros trabalhadores da família); tempo de relacionamento atual (número total de meses de união, ou seja, o casal habita o mesmo lar no momento da entrevista, independente do estado civil); uso de drogas psicoativas (drogas prescritas por clínicos ou psiquiatras com finalidade de tratar quadros psiquiátricos, em particular depressão); drogas ilícitas (uso de maconha, cocaína, crack); tempo de pós-parto (tempo decorrido entre o dia do parto e o dia da entrevista).

Para o rastreamento dos sintomas depressivos pós-parto foi utilizada a versão brasileira da Escala de Depressão Pós-Parto de Edimburgo (EPDS), instrumento de autorregistro composto de 10 enunciados cujas opções são pontuadas ( 0 a 3 ) de acordo com a presença ou a intensidade do sintoma, pelo qual considera-se depressão, escore igual ou superior a 12.3

As alterações tireoidianas foram definidas como: hipertireoidismo clínico (TSH baixo e T4 livre elevado); hipertireoidismo subclínico (TSH baixo e T4 livre normal); hipotireoidismo clínico (TSH alto e T4 livre baixo); hipotireoidismo subclínico (TSH elevado e T4 livre normal); anticorpos anti-peroxidase positivo (níveis séricos superiores a 60 $\mathrm{mUI} / \mathrm{mL}$ ).

Para tal foram dosados, por ensaios de quimioluminescência, os níveis séricos dos hormônios tireoestimulante (TSH ADVIA Centaur, Bayer HealthCare LLC. Valores de referência: 0.35 a 5.50 $\mathrm{uUI} / \mathrm{mL}$ ) e tiroxina livre (FrT4 ADVIA Centaur, Bayer HealthCare LLC. Valores de referência: 0.70 a $2.00 \mathrm{ng} / \mathrm{dL})$, e dos anticorpos antiperoxidase (IMMULITTE 2000 Anti-TPO EURO/DPC. Valor de referência: inferior a $60 \mathrm{UI} / \mathrm{mL}$ )

Os exames laboratoriais foram realizados em parceria com o Laboratório Pretti de Análises Clínicas, sem ônus para o pesquisador, que em nenhum momento recebeu verba pela execução do estudo.
Todas as informações coletadas foram inseridas no SPSS 11.5 (Statistical Package for the Social Sciences for Personal Computer), versão para Windows. Aos dados coletados, foram aplicadas técnicas de análise descritiva para caracterizar a amostra estudada. Para testar a associação entre as doenças estudadas, foi aplicado o teste $\chi^{2}$. Adotou-se nível de significância de 5\%.

$\mathrm{O}$ projeto de pesquisa e o termo de consentimento livre e esclarecido foram aprovados pelo Comitê de Ética em Pesquisa da Universidade Federal de São Paulo sob o número 0941/04.

\section{Resultados}

A amostra estudada incluiu 292 mulheres que se encontravam entre 31 e 180 dias após o parto (média de 2,7 meses), com média de idade materna de 24,7 anos (DP 6,054), predomínio de solteiras $(57,2 \%)$ e brancas $(49 \%)$. Independente do estado civil, a maioria $(95,9 \%)$ tinha parceiro sexual estável, com quem vivia maritalmente. $\mathrm{O}$ tempo médio de relacionamento do casal foi de 55,08 meses, o que equivale a 4,5 anos de convivência. Quanto ao nível de escolaridade, $89(30,5 \%)$ completaram o ensino médio, $42(14,4 \%)$ completaram o ensino fundamental e $6(2,1 \%)$, o ensino superior. Em 15,8\% a renda familiar era inferior a um salário mínimo, em $36 \%$ entre um e dois salários mínimos, 31,5\% de dois a cinco salários, $15,8 \%$ superior a cinco salários e $1 \%$ não soube informar. Em relação aos fatores associados ao comportamento e estilo de vida, $90,4 \%, 99,7 \%$ e $99,7 \%$ das participantes negavam o uso de tabaco, álcool e drogas, respectivamente. $\mathrm{O}$ perfil dos parceiros, também analisado, não diferiu das mulheres, com predomínio de homens jovens, com nível médio completo de escolaridade, não usuários de tabaco, álcool e drogas.

A prevalência de sintomas depressivos (EPDS: escore $\geq 12$ ) encontrada nesta amostra foi de $39,4 \%$.

Ao ser avaliada a função tireoidiana pela dosagem sérica dos níveis de TSH, T4 livre e antiTPO demonstrou-se a presença de alteração tireoidiana em 25 mulheres $(8,6 \%)$, das quais 9 (36\%) apresentaram sintomas depressivos concomitantemente. Neste grupo, as alterações tireoidianas observadas foram: cinco casos de hipertireidismo subclínico e anti-TPO (-), um com hipertireoidismo subclínico e anti-TPO (+) e três com anti-TPO(+) e níveis hormonais normais.

Entre as 267 pacientes $(91,4 \%)$ que não apresentaram alterações tireoidianas, 106 (40\%) apresentavam sintomas depressivos.

Não houve diferença estatisticamente signifi- 
cante na frequência de sintomas depressivos entre as pacientes com e sem alterações tireoidianas, indicando não haver associação entre depressão pós- parto e a alteração tireoidiana em nossa amostra populacional (Tabela 1).

Tabela 1

\begin{tabular}{|c|c|c|c|c|c|c|c|}
\hline \multirow[t]{3}{*}{ Alterações tireoidianas } & \multicolumn{4}{|c|}{ Depressão } & \multirow[t]{3}{*}{ Total } & \multirow[t]{3}{*}{$\chi^{2}$} & \multirow[t]{3}{*}{$p$} \\
\hline & \multicolumn{2}{|c|}{ Sim } & \multicolumn{2}{|c|}{ Não } & & & \\
\hline & $\mathrm{n}$ & $\%$ & $\mathrm{n}$ & $\%$ & & & \\
\hline Sim & 9 & 36 & 16 & 64 & 25 & 0.131 & 0.717 \\
\hline Não & 106 & 40 & 161 & 60 & 267 & & \\
\hline Total & 115 & 39 & 177 & 61 & 292 & & \\
\hline
\end{tabular}

\section{Discussão}

Sintomas psiquiátricos são comuns no ciclo gravídico-puerperal, especialmente no primeiro e no terceiro trimestres, e nos primeiros 30 dias após o parto, resultantes de alterações hormonais próprias do período e de mudanças no caráter social, na organização familiar, na autoimagem e na identidade femininas.

Embora não se disponha de parâmetros fisiológicos para avaliar as manifestações clínicas da depressão, escalas de avaliação são usadas para medir e caracterizar os sintomas, mesmo não podendo ser utilizadas como critério diagnóstico. 11

Neste estudo, optou-se por utilizar a Escala de Depressão Pós-Parto de Edimburgo como método de rastreamento de sintomas depressivos tendo por base o fato dessa escala ser instrumento de autoavaliação específico para o pós-parto, com sensibilidade e especificidade superiores a outros métodos disponíveis, tais como: Inventário de Beck, Escala de Montgomery-Asberg e Escala de Raskin, como relatado por Harris et al. 25 em estudo comparativo.

$\mathrm{Na}$ amostra do presente estudo foi constatada a prevalência de 39,4\% de depressão. Essa alta frequência de depressão pós-parto confirma ser essa doença um sério problema de Saúde Pública e alerta para importância da sensibilização e capacitação dos profissionais da área da saúde em identificar e prestar os primeiros cuidados em relação à assistência da puérpera, o que diminuiria as consequências que o quadro depressivo pode determinar sobre a família. Os resultados são semelhantes aos descritos por Da-Silva et al., ${ }^{2}$ Skasufka 26 e Cruz et al.27 que, utilizando o mesmo instrumento, demonstraram prevalência de $42,8 \%, 36,8 \%$ e $37,1 \%$, respectivamente.

Apesar dos resultados do presente estudo serem compatíveis com os estudos anteriormente citados, outras publicações nacionais demonstram menores taxas de prevalência de depressão pós-parto, variando de 13 a $19 \%, 3-5$ justificadas pelas diferenças metodológicas. A amostra analisada por Santos et al.,3 além de pequena (69 mulheres), foi composta por uma população feminina com renda familiar e grau de escolaridade superiores à população da presente investigação. Cury 4 avaliou somente mulheres que se encontravam no $10^{\circ}$ dia após o parto, período onde predomina o quadro de tristeza materna e não a depressão pós-parto, e utilizou como instrumento diagnóstico o Inventário de Beck, cuja sensibilidade e especificidade são inferiores à Escala de Edimburgo, por nós adotada.25 Já Moraes et al. 5 utilizaram a Escala de Hamilton, que enfatiza sintomas somáticos, podendo levar a conclusões enganosas quando é usada em pacientes com doenças médicas sistêmicas, em que a presença daqueles sintomas não necessariamente indica episódio depressivo. ${ }^{11,28}$

Os dados da literatura internacional são bastante divergentes ( 0 a $60 \%$ ) quando avaliam a prevalência de depressão pós-parto, fato justificado não somente pelas diferenças metodológicas, (definições do período pós-parto e instrumentos diagnósticos adotados), mas também pelas diferenças sociais, econômicas, culturais e genéticas das populações estudadas. 29

A prevalência de alteração tireoidiana na amostra total foi de $8,6 \%$, dados semelhantes aos encontrados por Barca et al.,30 em São Paulo, que apuraram prevalência de $13,3 \%$, e por Stagnaro-Green 17 que, em estudo de revisão, descreve prevalências que 
variam de 1,1 a $17,7 \%$, com média de 7,5\%.

Além da definição da alteração tireoidiana ter sido baseada na dosagem sérica dos hormônios TSH e T4 livre, optou-se também pela determinação dos níveis séricos dos anticorpos anti-peroxidase para definirmos a etiologia autoimune da disfunção tireoidiana encontrada, por ser a primeira alteração a surgir no desenvolvimento do hipotireoidismo secundário à tireoidite, 31 tendo sido classificado também como casos de alteração tireoidiana as mulheres que apresentaram anti-TPO $(+)$, mesmo na ausência de alterações dos níveis de TSH e T4 livre. Não foram dosados outros anticorpos tireoidianos por ser o anti-TPO o mais frequente, sensível e específico deles. 31,32

A alteração tireoidiana encontrada com mais frequência em nosso estudo foi o hipertireoidismo (12 casos) sendo sua incidência observada entre o final do segundo mês até o quarto mês pós-parto. Não foi afastada a possibilidade dessas pacientes terem evoluído para a fase hipotireoidea, que geralmente acontece depois da fase tireotóxica, a partir do quarto mês, caracterizando a forma clássica de tireoidite pós-parto. 20 O único caso de hipotireoidismo ocorreu no quinto mês pós-parto. Todos os níveis séricos positivos de anti-TPO, não acompanhados de alterações dos níveis hormonais, ocorreram até o terceiro mês pós-parto, podendo ser consequência da retomada da imunidade materna, anteriormente inibida pela gestação.

Embora existam algumas evidências de que discretas alterações tireoidianas predisponham à depressão, 33 não se conhece os verdadeiros mecanismos fisiopatológicos que unem essas duas morbidades. A semelhança de sintomas, principalmente do hipotireoidismo com quadros de depressão, é fator que dificulta o diagnóstico diferencial, e são escassos os estudos que têm avaliado a alteração tireoidiana como fator de risco para depressão, o que seria interessante, pois permitiria que um exame laboratorial funcionasse como rastreador de quadros depressivos no período pós-parto.

Tanto a depressão quanto as alterações tireoideanas são tidas como específicas do pós-parto quando se manifestam nos primeiros 12 meses após o parto, 17,20,34 ocorrendo simultaneamente com maior frequência do segundo ao quinto mês. $8,18,20$ Foi estabelecida, neste estudo, a inclusão de mães que se encontravam entre 31 e 180 dias pós-parto. Como a pesquisa foi desenvolvida na forma de estudo transversal, que analisa a prevalência pontual de um evento, nossa prevalência pode ter sido subestimada, ao considerar que no momento da coleta dos dados, algumas mães poderiam ainda não ter desenvolvido as doenças estudadas, ou poderiam tê-las desenvolvido anteriormente e evoluído para a remissão espontânea antes do diagnóstico. No entanto, cremos que o estudo até seis meses após o parto cubra a grande maioria dos casos, e, por outro, lado teríamos muita dificuldade de conseguir retorno das pacientes em períodos mais longos.

Neste estudo foi encontrada a amostra entre as mulheres com alterações tireoidianas, 36\% deprimidas, e nas sem alterações tireoidianas, $40 \%$ de deprimidas, não sendo possível concluir que a depressão seja decorrente da disfunção tireoidiana. Outros estudos com objetivo de correlacionar as alterações tireoidianas com a depressão pós-parto foram realizados. Pop et al.,23 em estudo prospectivo de 303 mulheres eutireoideas, acompanhadas entre a $32^{\mathrm{a}}$ semana de gestação e a $34^{\mathrm{a}}$ semana após o parto, observaram que $7 \%$ delas desenvolveram disfunção tireoidiana no pós-parto. Entre essas, 38\% tiveram quadro depressivo definido pelo Research Diagnostic Criteria (RDC), enquanto somente 9,5\% no grupo sem disfunção tireoidiana tiveram depressão. Apesar disso, há relato de que pacientes deprimidas nesse período não apresentam, necessariamente, disfunção tireoidiana. 35 Seeler et al.,36 com o objetivo de correlacionar quadros de depressão, ansiedade e mudança de humor com a disfunção tireoidiana, estudaram 292 mulheres no segundo ou terceiro dia pós-parto. Adotaram o Inventário de Beck para o diagnóstico dos distúrbios comportamentais, e a função tireoidiana foi avaliada por dosagem hormonal e identificação do anticorpo antimicrossomal. Observaram que a incidência do estado de ansiedade foi significativamente maior no grupo com anticorpo antimicrossomal positivo do que no grupo com anticorpo negativo. Quanto ao quadro depressivo e distúrbio do humor, não houve diferença significativa entre os grupos.

Embora seja descrito que a prevalência de sintomas depressivos é maior no hipotireoidismo do que no hipertireoidismo, 37 a amostra do presente estudo não permitiu confirmar essa premissa, o que pode ser explicado pela baixa prevalência de hipotireoidismo encontrada (um caso em mulher não deprimida), e pelos casos de hipertireoidismo terem se distribuído de forma igualitária entre os grupos de deprimidas (seis casos) e de não deprimidas (seis casos). 
O grande número de mulheres deprimidas em nossa amostra confirma que a depressão é problema sério de Saúde Pública também na região Metropolitana de Vitória, onde desenvolvemos nossa pesquisa. Sendo assim, é fundamental conhecer os múltiplos fatores de risco envolvidos na sua gênese e reconhecer que a sintomatologia dos quadros depressivos se confundem, sobremaneira, com mudanças comportamentais e emocionais sofridas pelas mães após o parto, ao mesmo tempo que se sobrepõem a comorbidades tireoidianas.

Este estudo não constatou que a manifestação das alterações tireoidianas nos primeiros seis meses após o parto seja marcador para o desenvolvimento da depressão, entretanto a elevada frequência de DPP aqui observada demonstra a importância do obstetra e demais profissionais não especializados em Saúde Mental na detecção precoce das formas clínicas mais brandas de depressão, tendo como auxílio diagnóstico instrumentos como a Escala de Depressão Pós-parto de Edimburgo, reconhecida mundialmente pela sua eficácia e praticidade. Desta maneira, adotar este questionário nas consultas pósparto como rotina assistencial poderia reduzir o impacto psicossocial negativo da depressão na vida da mulher e seus familiares.

\section{Referências}

1. Brockington I. Diagnosis and management of post-partum disorders: a review. World Psychiatr. 2004; 3: 89-95.

2. Da-Silva VA, Moraes-Santos AR, Carvalho MS, Martins MLP, Teixeira NA Prenatal and postnatal depression among low income Brazilian women. Braz J Med Biol Res. 1998; 31: 799-804.

3. Santos MFS, Martins FC, Pasquali L. Escalas de auto-avaliação de depressão pós-parto: estudo no Brasil. Rev Psiquiar Clin. [periódico online]. 1999; 26 (2). [Acessado em 21 abril 2005] Disponível em: http://www.hcnet.usp.br/ ipq/revista/r262/artigo(90).htm

4. Cury AF. Depressão puerperal: relação com eventos vitais estressantes, modo de enfrentamento e níveis do ácido 5 hidroxiindolacético [tese]. Departamento de Obstetrícia e Ginecologia da Faculdade de Medicina da Universidade de São Paulo; 2001.

5. Moraes IGS, Pinheiro RT, Silva RA, Horta BL, Sousa PLR, Faria AD. Prevalência da depressão pós-parto e fatores associados. Rev Saúde Pública. 2006, 40: 65-70.

6. Cox JL. Postnatal depression: a guide for health professionals. Edinburgh: Churchill Livingstone; 1986.

7. Stuart S, Couser G, Schilder K, O'Hara MW, Gorman L Postpartum anxiety and depression: onset and comorbidity in a community sample. J Nerv Ment Dis. 1998, 186: 4204.

8. O'Hara MW, Zekoski EM Postpartum depression: a comprehensive: review. In: Kumar R, Brockington IF, editors. Motherhood and mental illness. London: Oxford University Press; 1988. p.17-63.

9. APA (American Psychiatric Association). Diagnostic and statistical manual of mental disorder. 4. ed. Washington, DC; 2000.

10. Susman JL. Postpartum depressive disorders. J Fam Pract. 1996, 43: S17-S24.
11. Moreno AR, Moreno DH. Escalas de avaliação para depressão de Hamilton (HAM-D) e Montgomery-Asberg (MADRS). Rev Psiquiatr Clin. [periódico online]. 1998; 25 (4): 71-87. [acesso em: 21 abr 2005]. Disponível em: URL:http//hcnet.usp.br/ipq/revista/index.html.

12. Georgiopoulos AM, Bryan TL, Yawn BP, Houston MS, Rummans TA, Therneau TM. Population-based screening for postpartum depression. Obstet Gynecol. 1999, 93: 6537.

13. Beck CT. Predictors of postpartum depression. Nurs Res. 2001, 50: 275-85.

14. Piyasil V. Anxiety and depression in teenage mothers: a comparative study. J Med Assoc Thai. 1998, 81: 125-9.

15. Lopes AC, Del Porto JA. Transtornos afetivos e tiroidopatia. Psiquiatr Prat Méd. [periódico online]. 2000; 33 (3) [acesso em: 24 abr.2005]. Disponível em: http://www.unifesp.br/dpsiq/polbr/ppm/atu3_03.htm.

16. Boswell EB Anfinson TH Nemeroff CB. Depression associated with endocrine disorders. In: Robertson MM, Katona CLE, editors. Depression and physical illness. Chichester: Wiley; 1997. p. 256-92.

17. Stagnaro-Green A. Postpartum thyroiditis. Best Prac Res Clin Endocrinol Metabol. 2004; 18: 303-16.

18. Amino N, Mori H, Iwatani Y, Tanizawa O, Kawashima M, Tsuge I, Ibaragi K, Kumahara Y, Miyai K. High prevalence of transient post-partum thyrotoxicosis and hypothyroidism. N Engl J Med. 1982; 306: 849-52.

19. Goldman JM. Postpartum thyroid dysfunction. Arch Intern Med. 1986; 146: 1296-9.

20. Gerstein HC. How common is postpartum thyroiditis? A methodologic overview of the literature. Arch Intern Med. 1990; 150: 1397-400.

21. Pop VJM, de Rooy HAM, Vader HL, van der Heid D, van Son MM, Komproe IH. Microsomal antibodies during 
gestation in relation to postpartum thyroid dysfunction and depression. Acta Endocrinol. 1993, 129: 26-30.

22. Hall R. Pregnancy and autoimmune endocrine disease Baillière's Clin Endocrinol Metabol. 1995; 9: 137-55.

23. Pop VJ, de Rooy HA, Vader HL, van der Heide D, van Son M, Komproe IH, Essed GG, de Geus CA. Postpartum thyroid dysfunction and depression in an unselected population. N Engl J Med. 1991; 324: 1815-6.

24. Soares CN. Depressão puerperal, tensão pré-menstrual e depressão na menopausa. In: Lafer B, Almeida OP, Fraguás RJr, Miguel EC, editores. Depressão no ciclo da vida. Porto Alegre: Artmed; 2000. p. 144-56.

25. Harris B, Huckle P, Thomas R, Johns S, Fung H. The use of rating scales to identify post-natal depression. $\mathrm{Br} \mathrm{J}$ Psychiatry. 1989; 154: 813-7.

26. Skazufka, ET. Prevalência da depressão pós-parto e fatores associados em mães que deram à luz em um hospital universitário do município de São Paulo [dissertação]. São Paulo: Faculdade de Medicina da Universidade de São Paulo; 2000.

27. Cruz, EBS, Simões, GL, Cury, AF. Rastreamento da depressão pós-parto em mulheres atendidas pelo Programa de Saúde da Família. Rev Bras Ginecol Obstet. 2005; 27 : 181-8.

28. Blacker D. Psychiatric rating scales. In: Sadock B, Sadock V, editors. Kaplan \& Sadock's. Comprehensive textbook of psychiatry. 7. ed. Philadelphia: Lippincott Williams \& Wilkins; 2000. v. 1, p. 755-83.

29. Halbreich U, Karkun S. Cross-cultural and social diversity of prevalence of postpartum depression and depressive symptoms. J Affec Dis. 2006; 91: 97-111.

Recebido em 15 de outubro de 2008

Versão final apresentada em 12 de abril de 2009

Aprovado em 14 de maio de 2009
30. Barca MF, Knobel M, Tomimori E, Cardia MS, MedeirosNeto G. Prevalence and characteristics of postpartum thyroid dysfunction in Sao Paulo, Brazil. Clin Endocrinol. 2000; 53: 21-31.

31. Mariotti S, Caturegli P, Piccolo P, Barbesino G, Pinchera A. Antithyroid peroxidase autoantibodies in thyroid diseases. J Clin Endocrinol Metab. 1990; 71: 661-9.

32. Kasamatsu TS, Maciel RMB, Vieira JGH. Desenvolvimento e validação de um método imunofluorométrico para a pesquisa de anticorpos antiperoxidase tireoidiana no soro. Arq Bras Endocrinol Metab. 2002; 46: 167-72.

33. McCoy SJB, Beal JM, Payton ME, Stewart AL, DeMers AM, Watson GH. Postpartum thyroid measures and depressive symptomology: a pilot study. J Am Osteopath Assoc. 2008; 108: 503-7.

34. Evins G, Theofrastous JP, Galvin SL. Postpartum depression: a comparison of screening and routine clinical evaluation. Am J Obstet Gynecol. 2000; 182: 1080-2.

35. George AJ Wilson KCM. Beta-endorphin and puerperal psychiatric syntoms. Br J Pharmacol. 1983; 80: 493.

36. Seeler MJ, Christiansen K, Wegmann R, Bohnet HG. Personality markers physical complaints and microsomal thyroid antibody titer in postpartum women. Z Geburtshilfe Neonatol.1996; 200: 138-43.

37. Boswell EB, Anfinson TH, Nemeroff CB. Depression associated with endocrine disorders. In: Robertson MM, Kanota CLE, editors. Depression and physical illness. England: Wiley Chichester; 1997. p. 256-92. 